\title{
Metallographic and Fractographic Contributions to Understanding Environmentally Assisted Cracking
}

\author{
Stan Lynch $^{1}$ \\ ${ }^{1}$ Defence Science and Technology Organisation, Melbourne, Australia
}

Understanding environmentally assisted cracking - with a view to designing more-resistant materials and preventing failures - requires an awareness of processes occurring at and around crack tips on the meso-, micro-, and nano-scales in materials with well-characterised microstructures. A wide variety of metallographic, fractographic, and related experimental techniques, which are continuously being improved, are available to obtain the required information, and these techniques will be reviewed in the presentation. The most logical approach to understanding environmentally assisted cracking is to study single-phase single crystals in 'simple' environments in the first instance, and then build up the level of complexity towards studies of commercial, multiphase materials in environments where numerous interactions are possible $[1,2]$.

Optical microscopy, scanning electron microscopy (SEM), and transmission electron microscopy (TEM) (of fracture-surface replicas and thin foils), are the main techniques used to characterise microstructures, slip distributions around cracks, and fracture-surface appearance, as illustrated in figure 1 for an aged Al-Zn-Mg single crystal cracked rapidly in distilled water (where environmental interactions were 'simple' in that there was only time for hydrogen adsorption at crack tips without hydrogen diffusion ahead of cracks). Slip distributions around cracks in the interior of specimens can be determined by heat treating to decorate dislocations with solute atoms or precipitates, then sectioning, polishing, etching, and using optical microscopy. Alternatively, dislocation arrangements can be viewed directly by thin-foil TEM of cracked specimens, using focused-ion-beam preparation techniques. In-situ TEM of stressed thin foils have also been used to directly observe dislocation activity (and nano-void formation) during cracking in inert and hydrogen-gas environments [1,2].

Additional techniques to characterise cracking/microstructures include (i) Electron-Back-Scattered Diffraction (EBSD) to estimate strains and, for intergranular cracking, determine grain-boundary misorientations that promote or inhibit cracking, (ii) Scanning-Probe Microscopy, e.g. Atomic Force Microscopy (AFM), and Scanning Tunnelling Microscopy (STM), with the latter also providing information on the effects of adsorbates on surface structure and bonding, (iii) X-ray techniques including 3-D tomography, (iv) Auger Electron Spectroscopy (AES) of intergranular fracture surfaces to determine if segregants had been present, and (v) electrochemical potential measurements on fracture surfaces $[1,2]$.

The characteristics of environmentally induced cleavage-like cracking determined by the above techniques (and illustrated in figure 1 for an $\mathrm{Al}$ alloy in water) have also been observed for $\mathrm{Al}$ alloys in liquid metals, and a number of other materials in liquid-metal, hydrogen-gas, and aqueous environments. The observations, along with other evidence, support an Adsorption-Induced Dislocation-Emission (AIDE)/Void-Coalescence mechanism for liquid-metal embrittlement (LME) (where only adsorption can generally occur), hydrogen embrittlement (HE), and stress-corrosion cracking (SCC) in materials exhibiting the features shown in figure 1. The AIDE mechanism, illustrated 
in figure 2 for cleavage-like cracking, may also be applicable to intergranular LME, HE, and SCC in some (but not all) systems [1,2].

\section{References:}

[1] S.P. Lynch, Metallographic and fractographic techniques for characterising and understanding hydrogen-assisted cracking, in "Gaseous hydrogen embrittlement of materials in energy technologies", Vol. 1, ed. R.P. Gangloff and B.P. Somerday, Woodhead, Cambridge, UK, 2012, pp. 274-346, and references therein.

[2] S.P. Lynch, Mechanistic and fractographic aspects of stress-corrosion cracking, in "Stress corrosion cracking: Theory and practice", ed. V.S. Raja and T. Shoji, Woodhead, Cambridge, UK, 2011, pp. 3-89, and references therein.
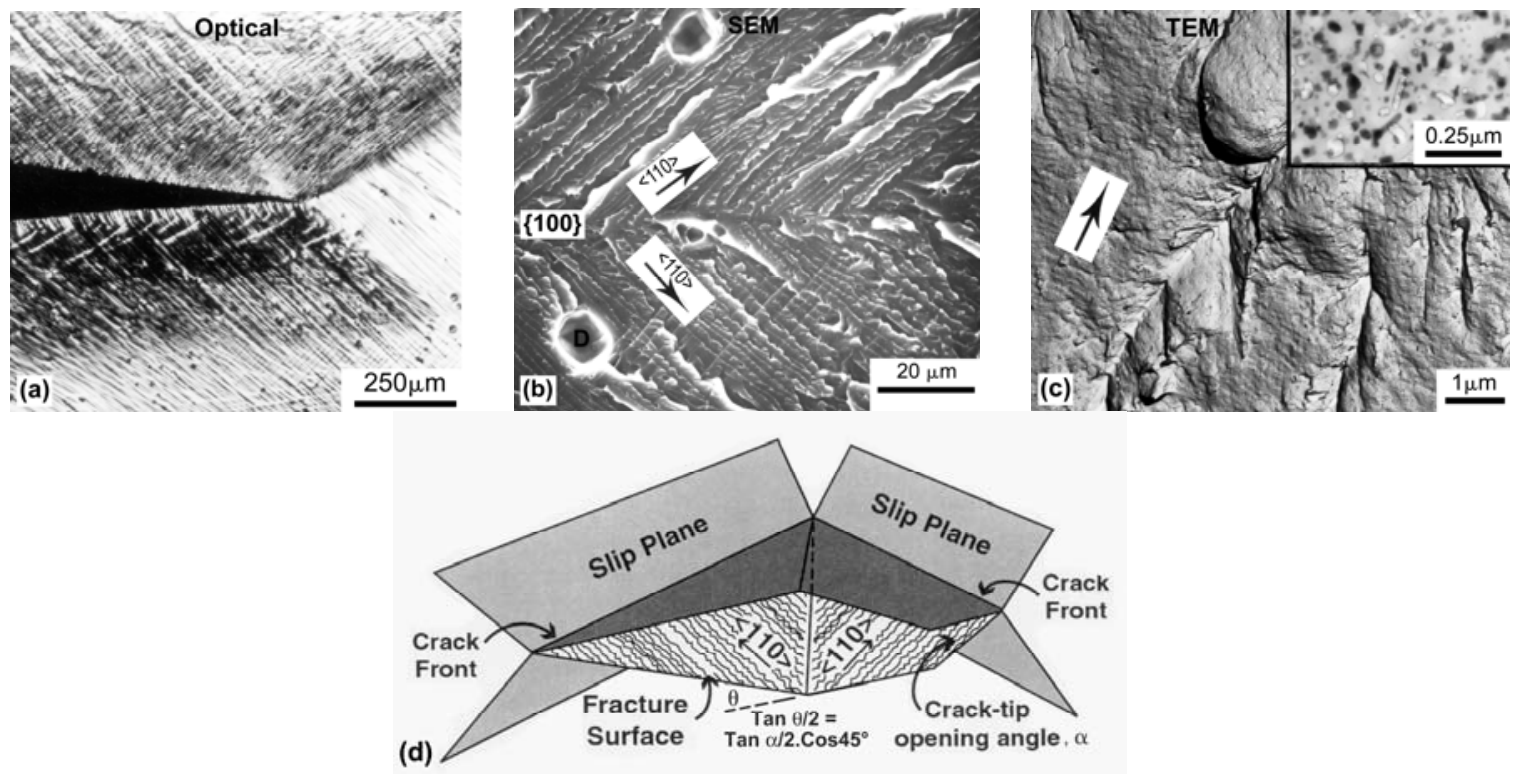

Figure 1. Characteristics of environmentally assisted cracking in aged $\mathrm{Al}-\mathrm{Zn}-\mathrm{Mg}$ single crystals after rapid crack growth $(\sim 10 \mathrm{~mm} / \mathrm{s})$ in distilled water: (a) Optical micrograph showing slip on pre-polished side surface, (b) SEM of fracture surface showing herringbone pattern of steps/tear ridges, along with isolated large dimples (D), (c) TEM of replica of fracture surface showing shallow, nano-scale dimples associated with voids formed ahead of the crack around age-hardening precipitates (see inset), and (d) schematic diagram illustrating crack-plane/slip-plane geometry based on the observations [1,2].

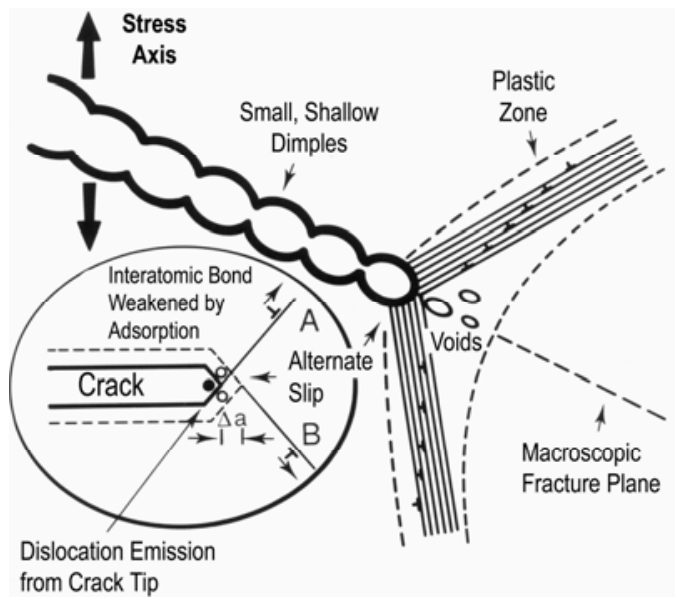

Figure 2. Schematic diagram illustrating the Adsorption-Induced Dislocation-Emission (AIDE)/Void-Coalescence model for LME, HE, and SCC, which can account for the above observations $[1,2]$. 